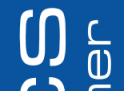 \\ Interference of Two Point Sources Using Smartphones
}

Cite as: Phys. Teach. 59, 709 (2021); https://doi.org/10.1119/10.0007409

Published Online: 19 November 2021

\section{Giovanni Organtini}

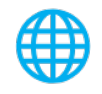

\section{ARTICLES YOU MAY BE INTERESTED IN}

A Student Experiment About Rainbows: Ray Tracing Through an Acrylic Cylinder

The Physics Teacher 59, 692 (2021); https://doi.org/10.1119/10.0007394

The Electric Monochord: A Musical Demonstration About Electromagnetic Induction The Physics Teacher 59, 694 (2021); https://doi.org/10.1119/10.0007395

The Induction Coil

The Physics Teacher 59, 689 (2021); https://doi.org/10.1119/10.0007393

AMERICAN PThe sics
JOURNAL PHYSICS
of PHYSICS

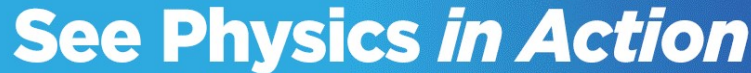

VIDEO ABSTRACTS TO VISUALIZE POPULAR ARTICLES
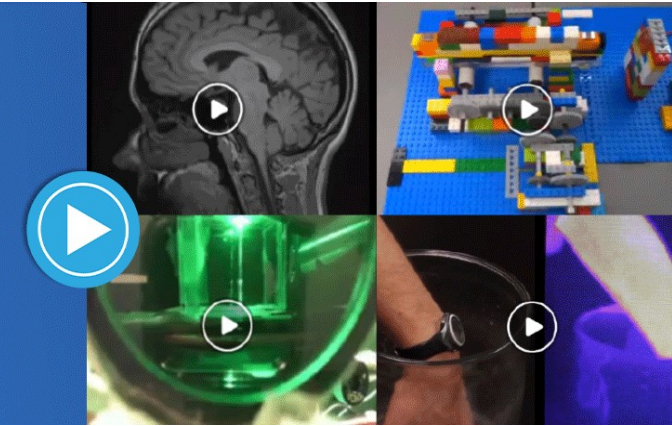


\section{Interference of Two Point Sources Using Smartphones}

Giovanni Organtini, Sapienza Università di Roma, Roma, Italy

A simple experimental setup using a smartphone and a pair of speakers is presented to perform an accurate experiment on interference of two point sources. The proposed experiment allows simple but interesting measurements to be done to introduce students to interference and diffraction phenomena. As such, the experiment effectively introduces the classical Young's double-slit interference experiment, and can be used as a replacement for the classical ripple tank.

\section{Introduction}

Among the crucial experiments in physics history, Young's double-slit experiment ${ }^{1}$ is certainly one of the most relevant in many aspects. It was simple, yet fundamental for the determination of the nature of light and it was an instructive example about how science makes progress, subverting the theories of predecessors like Newton, irrespective of their reputation. In fact, Newton was in favor of a corpuscular theory of light that was proven to be rrong $^{2}$ by Young's experiment. ${ }^{3}$

For this reason, its execution in classrooms is frequent in various forms. The experiment is, in fact, conceptually composed of two steps: the first consists of producing two inphase sources, the second demonstrating that the two sources interfere. An experimental demonstration is usually made using a ripple tank: a relatively expensive and complex setup. As a consequence, students can only look at the experiment and do not really perform it. Moreover, drawing the students' attention to the pattern observed when the waves emerge from the slits is difficult due to the size of the apparatus, and due to the fact that the pattern is often barely visible, such that it is advisable to freeze the pattern using stroboscopic lights. The latter, in turn, adds an additional layer to the interpretation of the experiment, adds some cost to it, and sometimes may lead to safety issues because of photo-induced epilepsy.

On the other hand, demonstrating that a beam of plane waves generates in-phase circular waves after passing narrow gaps on a barrier is much simpler. In this paper we describe an experiment made with acoustic waves leading to an interference pattern similar to those obtained with light or ripple tanks, when performing Young's experiment. On the other hand, even Thomas Young in his papers often recalled the analogy between the two. ${ }^{4,5}$

Thanks to the availability and the ubiquity of tools such as smartphones, letting each student perform their own experiment is now possible. Many experiments are possible with this tool. ${ }^{6,7}$ In this paper we show a possible setup for a "double-slit" experiment that only needs a smartphone and a pair of speakers connected to a source that can either be another smartphone or a computer.

We begin describing the setup used by us to take measurements, as well as the data taking. Data collected during the measurements are then analyzed in the next section both in a simplified way, suitable for high school students, and in a more professional way, suitable for undergraduate students.

\section{Experimental setup and data taking}

In a double-slit experiment, the two slits are needed as two in-phase sources of waves. Such a source of waves can be provided by a pair of speakers connected to a computer or to a smartphone. To perform the experiment one needs a powerful enough source of waves, hence speakers must be amplified; however, a modest amplification is more than enough and speakers with a power output between 1 and $5 \mathrm{~W}$ are perfectly suitable. We used a USB-powered pair of speakers connected to a computer.

The experiment consists of playing a sinusoidal tone with the speakers and detecting the intensity of the sound at various angles at some distance from the sources using a smartphone. The frequency of the sound must be such that it can be detected by the smartphone's microphone. On the other hand, it should be high enough to allow for various maxima and minima being detected.

As it is well known, the distance $x$ between two consecutive maxima or minima is given by

$$
x \simeq L \frac{\lambda}{d}=L \frac{c}{d f},
$$

where $L \gg d$ is the distance between the source and the detector, $\lambda$ the sound wavelength, $d$ the distance between the speakers, $c$ the speed of sound, and $f$ its frequency.

The distance between speakers can be of the order of $10 \mathrm{~cm}$, while the distance between the latter and the detector cannot be too large, for practical reasons. We assume it is on the order of $1 \mathrm{~m}$. The speed of sound $c \simeq 300 \mathrm{~m} / \mathrm{s}$; hence, to have $x \simeq 10 \mathrm{~cm}$ we need

$$
f \simeq L \frac{c}{d x}=30 \mathrm{kHz}
$$

We then placed our speakers $32.0 \pm 0.1 \mathrm{~cm}$ apart and fixed a ruler parallel to the line connecting the two speakers at 1.120 $\pm 0.001 \mathrm{~m}$ from them. The ruler is used both as a guide along which the smartphone can be translated and as a tool to measure the distance of the smartphone with respect to the normal to the line connecting the speakers. We chose to make measurements at $f=15 \mathrm{kHz}$, which is an audible frequency, high enough and not too unpleasant. The tone was generated using the http://onlinetonegenerator.com/ site.

The experiment was done in a small office. That makes it possible, in principle, to detect echoes and reverberation effects. However, we found that they were not too large and do not have a big impact on the results, in fact. Another possible disturbing effect can be the fact that the sound wave travels on 
the surface of the table on which the experiment is performed. The results we obtained show that this effect is not disturbing either. In fact, our setup was, like running the experiment, rather rough on purpose, and some choices are a bit too extreme, in order to mimic the way an inexperienced student would have chosen. This shows how good results can be, even if the parameters of the experiment are not optimized.

The smartphone was placed in contact with the ruler. Its position is determined finding the mark on the ruler corresponding to the position of its microphone, identified as a small hole on its lower edge. The starting position, corresponding to the intersection point between the line identified by the ruler and its perpendicular passing through the center of the segment connecting the two speakers, is defined as $x=0$.

We used phyphox ${ }^{7}$ to make measurements, which is an open source app for smartphone designed by RWTH Aachen and freely available for both iOS and Android phones. It allows to use onboard sensors to perform physics experiments. It collects raw data for each sensor found in the phone and makes it possible to save those data on external storage, as well as providing online calculations to extract physics data from the measurements. The source of the tone used to perform the experiment can even be a second smartphone with phyphox, the app providing a tone generator function, too.

The smartphone's microphone is one of the sensors exploited by phyphox. We used the audioscope function to record the waveform of the sound collected by the microphone during a time interval whose duration can be adjusted between 1 and $500 \mathrm{~ms}$. We collected data for $10 \mathrm{~ms}$ at each position between $x=0$ and $x=29.0 \pm 0.1 \mathrm{~cm}$.

In the audioscope, the amplitude of the recorded sound is plotted against time on the phone's screen. The plot can be expanded to full screen, and the coordinates of selected points can be read directly on the screen. When the frequency of the sound is rather high, as in this case, aliasing may distort the shape of the wave, so we prefer to export data to a computer for further analysis. Aliasing can be mitigated reducing the frequency, at the price of increasing $x$. On the other hand, using a computer to perform the analysis has the advantage of making students practice the use of such a tool for scientific investigation. Another advantage of phyphox over similar tools is, in fact, that teachers can choose among many different analysis schemes, depending on the class, fostering the acquisition of different skills.

For each measurement, phyphox provides a comma separated value file with two columns: time (in ms) and amplitude (in arbitrary units). The whole process (positioning the smartphone, starting data taking, saving data) is very fast, indeed, and the full set of data could be collected in less than half an hour.

\section{Data analysis}

In this section we show the results obtained with the setup described above in both a simplified and detailed way. The first method is suitable for high school students, while the second is more suitable for undergraduate students. It is worth noting that the simplified version is not the simplest possible: it is, however, simple enough to be performed by a student, yet complex enough to involve the usage of a computer and to reduce systematics.

\section{Simplified analysis}

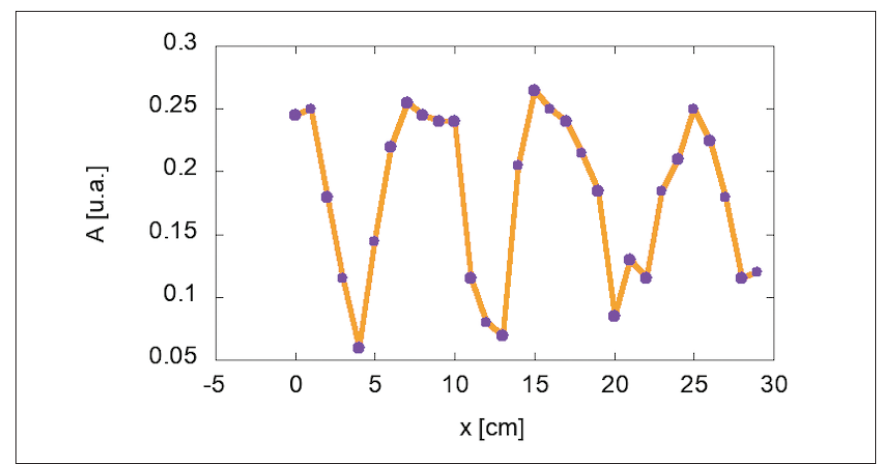

Fig. 1. The maximum amplitude of the audio signal recorded with a smartphone as a function of its position.

In the simplified analysis we just look for the maximum amplitude in the file. A plot of the maximum as a function of $x$ is shown in Fig. 1. Maxima and minima are clearly visible. Just identifying the relative extrema by eye, one can determine their position as $x=\{1,4,7,13,15,20,25,28\} \mathrm{cm}$. Correspondingly, the differences between adjacent points are $\Delta=3$, $3,6,2,5,5,3 \mathrm{~cm}$, whose average is $\Delta=3.9 \mathrm{~cm}$, with a standard deviation of about $1.5 \mathrm{~cm}$. The expected value for $\Delta$ is

$$
\Delta \simeq \frac{1}{2} \frac{c L}{f d} \simeq 4 \mathrm{~cm},
$$

very close to the experimental value, despite the crude approximations done in both the theoretical and experimental data analysis.

It must be noted that adopting more clever strategies may lead to better results (e.g., using only the minima or only the maxima identified as the centers of the peaks). Adopting the above, non-optimized approach, in our opinion, leads to a much improved self-efficacy in students. Experiencing the effects of measurement errors, they do not get discouraged when they obtain, in a single measurement, values that appear to significantly differ from their own expectations.

Of course, the experiment can be used both as a verification of the predictions or as a starting point to analyze wave mechanics and interference by two sources.

\section{In-depth analysis}

The frequency of the wave can be obtained by the measurements itself. Most modern smartphones sample the signal at high enough frequency. The latter can be easily obtained experimentally, observing the time difference $\Delta t$ between successive samples. Our smartphone had a sampling rate of about $50 \mathrm{~ms}^{-1}$, given that $\Delta t \simeq 0.02 \mathrm{~ms}$, compatible with $48 \mathrm{kHz}$, one of the commonly used sampling rates on smartphones, and to be compared with a period of $0.07 \mathrm{~ms}$ of the generated tone. From a fit to the data with the function $I(t)=A \sin (2 \pi f t$ $+\phi)$, we can get the amplitude $A$, the frequency $f$, and the 


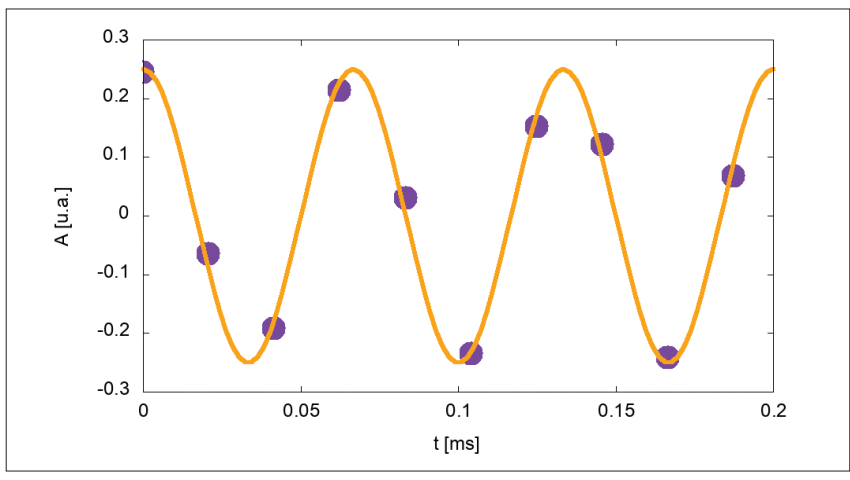

Fig. 2. Fit to the waveform made on a sample of the data recorded by the smartphone in one experiment.

phase $\phi$. The average frequency from the 30 measurements is $15000 \pm 1 \mathrm{~Hz}$, as expected. The expected intensity distribution is ${ }^{9}$ :

$$
I(x)=2 I_{0}^{2}\left[1+\cos \left(\frac{2 \pi f}{c} d \sin \theta\right)\right],
$$

where $\theta$ is defined such that $L \tan \theta=x$. From Fig. 1 , it can be noted that the distribution is affected by an increasing level of noise with increasing $x$, while the maximum intensity remains capped around 0.25 and that is the value at which the maximum amplitude is always shown in arbitrary units by phyphox. Fitting data to

$I(x)=2 I_{0}^{2}\left[1+\cos \left(\frac{2 \pi f}{c} d \sin \left(\tan ^{-1}\left(\frac{x}{L}\right)\right)\right)\right]+m x+q$

with $I_{0}, m, q$, and $f$ as free parameters, we found that the first minimum is at

$$
\Delta=\frac{c L}{2 f d}=4.05 \pm 0.03 \mathrm{~cm}
$$

for $f=14085 \pm 104 \mathrm{~Hz}$, compatible with the chosen frequency. The result is shown in Fig. 3.

Despite the quality of the fit not being high, due to relatively large systematic errors in the determination of the amplitude at each point, the results are in good agreement

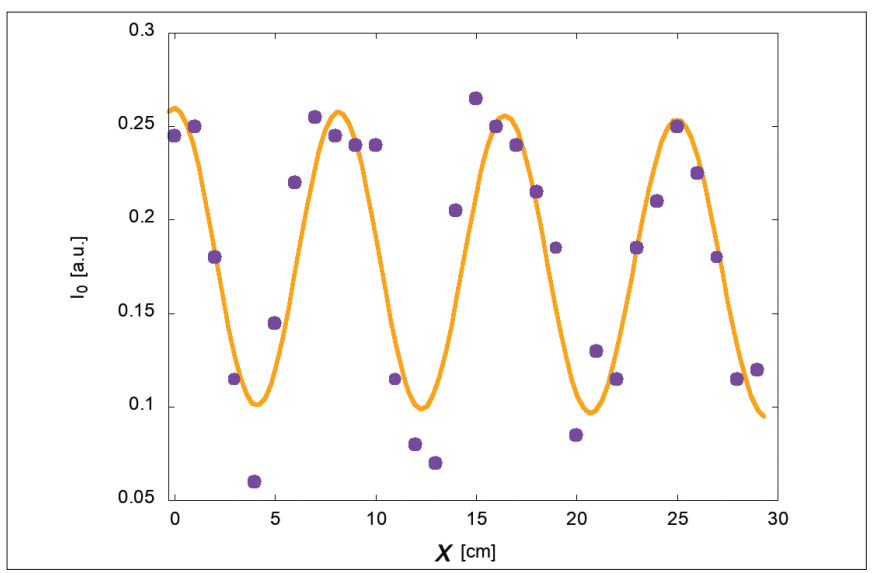

Fig. 3. Intensity of the sound as a function of the displacement of the microphone. Dots represent experimental data, while the curve is the fit result. with expectations, well beyond what we could anticipate from the fact that we deliberately made the measurements in a very crude way and without much care, as explained in the previous section.

\section{Summary}

An experiment is presented using a smartphone equipped with phyphox and a pair of speakers, aiming at studying the interference pattern emerging from the sum of the in-phase sound waves produced by the speakers. Data analysis, conducted both in simplified and detailed ways, allows a precise determination of all the characteristics predicted by the theory. The setup is simple enough to allow each single student to perform his/her own experiment at home, providing a solid ground for understanding Young's experiment on light to determine its wave nature and the corresponding frequency.

\section{Acknowledgment}

The author wishes to thank Prof. Egidio Longo for useful discussions.

\section{References}

1. Thomas Young, "Bakerian Lecture: Experiments and calculations relative to physical optics," Philos. Trans. R. Soc. London 94, 1-16 (1804).

2. In fact, the meaning of the adjective "wrong" should be discussed at length. Here, we assume that it means false under the circumstances in which Young's experiment was done.

3. Alain Aspect, "From Huygens waves to Einstein's photons: Weird light," C. R. Physique 18, 498-503 (2017).

4. Thomas Young, "Bakerian Lecture: On the theory of light and colours," Philos. Trans. R. Soc. London 92, 12-48 (1801).

5. Thomas Young, "Outlines of experiments and inquiries respecting sound and light," Philos. Trans. R. Soc. London 90, 106-150 (1800).

6. For a very general introduction, see, e.g., J. Kuhn, "Analyzing acoustic phenomena with a smartphone microphone," Phys. Teach. 51, 118 (Feb. 2013).

7. An example of activity that can be done using smartphones is, e.g., J. Kuhn, "Analyzing the acoustic beat with mobile devices," Phys. Teach. 52, 248 (April 2014).

8. S. Staacks, S. Hutz, H. Heinke, and C. Stampfer, "Advanced tools for smartphone-based experiments: phyphox," Phys. Educ. 53 (4), 045009 (2018).

9. R. Feynman, B. Leighton, and M. Sands, The Feynman Lectures on Physics (Addison-Wesley, Reading, MA, 1963). 